\title{
Impact of way of life and environment on the prevalence of Chlamydia felis in cats as potentional sources of infection for humans
}

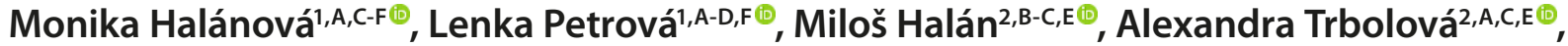

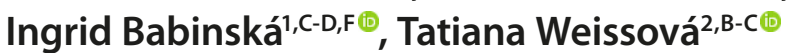 \\ ${ }^{1}$ Faculty of Medicine, Pavol Jozef Šafárik University, Košice, Slovakia \\ ${ }^{2}$ University of Veterinary Medicine and Pharmacy, Košice, Slovakia \\ A - Research concept and design, B - Collection and/or assembly of data, C - Data analysis and interpretation, \\ $D$ - Writing the article, E - Critical revision of the article, F - Final approval of article
}

Halánová M, Petrová L, Halán M, Trbolová A, Babinská I, Weissová T. Impact of way of life and environment on the prevalence of Chlamydia felis in cats as potentional sources of infection for humans. Ann Agric Environ Med. 2019; 26(2): 222-226. doi: 10.26444/aaem/100655

\begin{abstract}
Introduction and objectives. Chlamydia (C.) felis can cause infection which may be associated with conjunctivitis and/or respiratory tract disease, particularly in kittens, but could also be the cause of the disease in adult cats. Infection is more common in multi-cat environments. The zoonotic potential of $C$. felis appears low, but exposure to this microorganism is possible by handling the affected cats, by contact with their aerosol, and also via fomites.

Materials and method. In the study, 140 cats of various breeds from Košice region in Slovakia were studied. Conjunctival samples were obtained from 71 clinically healthy cats (50.7\%) and 69 cats with clinical signs of conjunctivitis and upper respiratory tract impairment (49.3\%). Cats were divided into 4 groups according to breed and type of environment in which they lived. In the $1^{\text {st }}$ group were cats kept inside only $(n=33)$, in the $2^{\text {nd }}$ group, free-roaming cats $(n=50)$, the $3^{\text {rd }}$ group comprised stray cats, taken from the streets $(n=28)$, and the $4^{\text {th }}$ group included cats kept in shelters or deposit devices $(n=29)$. Molecular method PCR and DNA sequencing was used as the diagnostic method.

Results. Overall positivity was $17.1 \%$. Of the 24 positive cats, the highest positivity was detected in the population of stray cats (35.7\%) and shelter cats (31\%). In the group of free-roaming cats, $10 \%$ had positivity. No positive animals were detected in the group of cats kept inside only. It was also found that the risk of $C$. felis in cats with clinical signs of disease was more than 7-fold higher than in cats without clinical signs of conjunctivitis and respiratory tract.

Conclusion. The obtained results show that cats, especially stray and shelter cats, can be important sources of feline chlamydiosis, and due to their close contact with people they can present a risk for transmission.
\end{abstract}

\section{Key words}

Chlamydia felis, cat, prevalence, zoonosis, PCR, public health

\section{INTRODUCTION}

The interaction between human and animal health is not a new phenomenon. However, the scope, scale, and worldwide impact of zoonoses we are facing today have no historical precedent. Of the 1,415 identified infectious pathogens for humans 868 (61\%) have a zoonotic character [1], and these diseases have an increasing trend, not only in developing but also in developed countries. The ecological changes caused due to the destruction of the natural environment increase the exposure of animals and humans to the vectors and reservoirs of unknown pathogenic microorganisms. In addition to this, weak public health systems, other natural disasters, absence of better surveillance and controlling systems against the disease and unhygienic living conditions are the main factors for the emergence and re-emergence of the bacterial zoonotic diseases in many countries worldwide [2]. The rapid detection and response to diagnostic diseases is crucial, because on many occasions there is an unobserved spreading and infiltration of diseases to new territorial units,

Address for correspondence: Lenka Petrová, Pavol Jozef Šafárik University in Košice, Faculty of Medicine, Košice, Slovakia

e-mail: lenka.petrova@upjs.sk

Received: 05.11.2018; accepted: 11.12.2018; first published: 09.01.2019 especially in the case of emerging, respectively, re-emerging diseases.

One of the most common microorganisms in nature which have a zoonotic character are chlamydiae from the family Chlamydiaceae. Chlamydiaceae has only a single genus, Chlamydia that comprises 12 species [3, 4], including Chlamydia felis. Chlamydia felis is an obligate intracellular bacterium that seems to have a predilection for conjunctival epithelial cells, and is the most common cause of feline chlamydiosis and different diseases in humans.

Feline chlamydiosis is the most common cause of pneumonia and acute or chronic conjunctivitis, particularly in kittens $[5,6,7]$, but could also be the cause of the disease in adult cats [8]. Clinical signs of infection with $C$. felis in cats are sneezing, transient fever, inappetence, weight lost, nasal discharge, vaginal discharge and lethargy. Chlamydial disease may be complicated by coinfection with other microorganisms. Feline chlamydiosis is more common in multi-cat environments, particularly breeding catteries where animals share a common space [9].

C. felis spreads through direct contact between cats, and cats and humans. Transmission between cats is due to direct contact with infectious eye secretions. This pathogen is highly unstable outside the host and remains infectious for a short time in the environment. 
The zoonotic potential of these bacteria seems to be low; however, $C$. felis may be exposed to the manipulation of infected cats. In particular, cat owners and employees working with cats (veterinarians, breeders'staff) are at increased risk, especially where poor hygiene conditions are present. The risk of zoonotic transmission is likely to be greater in immunocompromised individuals $[10,11,12$, $13,14]$. In general, acute conjunctivitis is a frequent human condition, with an estimated six million cases per year in the USA, and Chlamydiae being the cause in $1.8-5.6 \%$ of all cases [15]. However, only few human cases of $C$. felis conjunctivitis have been reported, despite its high incidence in cats and the large number of pet cats in society. Actual prevalence may be much higher because human infection is predominantly asymptomatic [16].

\section{OBJECTIVE}

Because the control of incidence of chlamydial infections between animals and humans is the key to a better understanding of the relationship between chlamydiae and their hosts, the aim of this study was to investigate the incidence of $C$. felis in cats, according to breeding and the type of environment in which they live, and to compare the incidence in cats with and without clinical signs of infection, and assess the zoonotic potential.

\section{MATERIALS AND METHOD}

Samples were collected from cats examined at the Department of Small Animals at the University of Veterinary Medicine and Pharmacy in Kosice, Slovakia, and various deposit centres (shelters) in Kosice, Slovakia. 140 cats of various breeds, aged from 1 month to 10 years were examined. Most of the examined cats were European shorthair cat breeds (ESH); lesser represented breeds were Maine Coon, British blue and Sphinx. Based on the type of environment, the animals were divided into 4 groups for examination:

1) cats kept inside human habitations, without possible contact with other cats and other animals. A requirement for inclusion in this group was also internal breeding. Total: 33 cats.

2) Free-roaming domestic cats, able to move around freely, and thus come into contact with other cats, respectively, animals. Total: 50 cats.

3) Stray cats taken from the streets and brought to the clinic for examination. Total: 28 cats.

4) Cats housed in shelters (so-called deposit facilities), characterized by a high concentration of animals. Total: 29 cats.

In each of the 4 groups, the cats were also divided on the basis of clinical signs of chlamydial infection. A total of 140 cats, 69 had clinical signs and the other 71 were without any pathological finding. None of the examined cats were vaccinated against chlamydia infection.

After administration of local anesthetic into the eye, samples were taken with a sterile cotton swab rolled deep into conjunctival sac to capture the biggest number of epithelial cells of the conjunctiva. Immediately after sampling from each eye, the cotton swab was macerated in tubes filled with
$300 \mu \mathrm{l}$ saline solution for 60 seconds. Samples thus obtained were stored at $-80^{\circ} \mathrm{C}$ until testing.

Molecular analysis. For isolation of the DNA, samples resulting from mixing $150 \mu \mathrm{l}$ samples from both eyes were used. DNA isolation was carried out according to the manufacturer's instructions using a commercial diagnostic kit DNA-Sorb/AM (Nucleic Acid Extraction Kit, AmpliSens, Federal State Institution of Science, Moscow, Russia). In cases where the amplification was not been carried out immediately, the samples were stored at $-20^{\circ} \mathrm{C}$ until their next use. Positive specimens for Chlamydiaceae spp. were subsequently amplified using a set of primers U23Fa (5'-GAT GCC TTG GCA TTG ATA GGC GAT GAA GGA-3`) and 23 SIGR (5'-TGG CTC ATC ATG CAA AAG GCA-3`) that amplify domain I of $23 \mathrm{~S}$ rRNA gene. The reaction mixture for one reaction was prepared by mixing $0.2 \mu \mathrm{l} 10 \mathrm{pmol}$ of primer $\mathrm{U} 23 \mathrm{Fa}, 0.2 \mu \mathrm{l} 10 \mathrm{pmol}$ of primer 23 SIGR, $9.6 \mu \mathrm{l}$ of purified water, $4 \mu \mathrm{l}$ of Taq polymerase (HOT FIREPol Blend Master Mix, Solis BioDyne, Estonia), and $6 \mu$ of template (isolated DNA). Electrophoresis was carried out for 20 minutes at $300 \mathrm{~V}$. PCR products were analysed by electrophoresis of $10 \mu \mathrm{l}$ of each $20 \mu \mathrm{l}$ reaction mixture on a $1.5 \%$ agarose gel stained using GoldView stain (SBS Genetech, China) and visualized fragments were compared with the positive control and the 100 bp DNA ladder.

Positive products Chlamydiaceae spp. were compared against the positive control for the length of $600 \mathrm{bp}$ under UV light at a wavelength of $254 \mathrm{~nm}$. The identity of the obtained sequences was examined by a BLAST search [17].

DNA sequence alignments and phylogenetic analysis were conducted using software MEGA5. Phylogenetic trees were created using alignments performed with the BioEditSequence Aligment Editor as a distance method and NJ (neighbour joining) as the tree construction method. All ambiguous positions were removed from each sequence pair. The reliability of branches in trees was assessed using bootstrap analysis with 1,000 pseudoreplicates, with values above $70 \%$ being noted.

Analysis of sequences for constructing the phylogenetic tree also included 9 reference strains, the partial sequence of the 23S rRNA gene of different chlamydial species from the Gene Bank database, C. felis FP Cello (U68458), C. felis VR 120 (U68457), C. pneumoniae (U68424), C. suis (U68420), C. muridarum (U68436), C. pecorum (U68439), C. avium (NR_121988), C. abortus (U68444), C. caviae (NR_076195), and Pirellula marina (AF 245367) was used as the outgroup.

Statistical analysis. Basic descriptive statistics were used for the analysis of the obtained results. To compare the incidence of chlamydial infections between the groups, a calculation of the relative risk (RR) and their 95 percent confidence intervals (95\% CI) was performed and estimated for the occurrence of $C$. felis infection. All tests were considered significant at $\mathrm{p}<0.05$.

\section{RESULTS}

Determination of the prevalence of $C$. felis infection in the population of cats in Slovakia and its comparison with stray, domestic cats and cats kept in shelters. Of the 140 cats examined by PCR, Chlamydia spp. was detected in 
24 (17.1\%). After amplification and electrophoretic analysis, positive samples were sent for sequencing. By analyzing the sequences of the $23 \mathrm{~S}$ rRNA gene to construct the phylogenetic tree using 9 reference strains of different species of chlamydia, in all positive cases the species $C$. felis was confirmed (Fig. 1).

Comparison of occurrence of C. felis infection in cats depending on clinical signs and farming method. When comparing the occurrence of $C$. felis in cats with conjunctivitis and/or clinical signs of respiratory tract disease, compared with cats without clinical signs, $30.4 \%$ positive for cats with clinical signs were confirmed versus $4.2 \%$ positive for cats without clinical signs. When calculating the relative risk between these groups, it was found that the risk of $C$. felis in cats with clinical signs of disease was more than 7 -fold higher $(\mathrm{P}=0.0095)$ than in cats without clinical signs of conjunctivitis and respiratory tract infection (Tab. 1)

The most common clinical sign of chlamydial infection was conjunctivitis with prevalence of purulent discharge, chemosis, hyperaemia, blepharospasm and mucopurulent discharge.

When comparing the occurrence of chlamydia infection, according by the farming method, it was found that stray cats and cats from shelters and deposits $35.7 \%$ and $31 \%$, respectively) were infected most frequently. In the group of free-roaming cats, from the 50 examined, 5 were positive (positivity 10\%). None of the cats from the group kept strictly within human habitations were detected chlamydial infection (Tab. 2).

By comparing the relative risk, it was found that compared to cats strictly kept inside, free-roaming cats had more than a 7-fold risk of chlamydial infection, cats from shelters and deposits had this risk of more than 21-fold, and stray cats almost 25-fold (Tab. 2).

Table 1. Occurrence of $C$. felis depending on occurrence of clinical signs

\begin{tabular}{lccccc}
\hline \multirow{2}{*}{ Cats } & \multicolumn{2}{c}{$\begin{array}{c}\text { No. of } \\
\text { examinations }\end{array}$} & \multicolumn{2}{c}{$\begin{array}{c}\text { No. of positive } \\
\text { case }\end{array}$} & $\begin{array}{c}\text { Relative risk } \\
(95 \% \mathrm{Cl})\end{array}$ \\
\cline { 2 - 5 } & $\mathrm{N}$ & $\%$ & $\mathrm{~N}$ & $\%$ & \\
\hline with clinical signs & 69 & 49.3 & 21 & 30.4 & $\begin{array}{c}7.2 \\
(2.25-23.06)\end{array}$ \\
\hline whithout clinical signs & 71 & 50.7 & 3 & 4.2 & $1^{*}$ \\
\hline$\Sigma$ & 140 & 100 & 24 & 17.1 & \\
\hline
\end{tabular}

*1 1 - reference group

Table 2. Prevalence of $C$. felis in cats by groups

\begin{tabular}{|c|c|c|c|c|c|}
\hline \multirow[t]{2}{*}{ Group } & \multicolumn{2}{|c|}{$\begin{array}{c}\text { No. of } \\
\text { examinations }\end{array}$} & \multicolumn{2}{|c|}{$\begin{array}{c}\text { No. of } \\
\text { positive case }\end{array}$} & \multirow{2}{*}{$\begin{array}{l}\text { Relative risk } \\
\qquad(95 \% \mathrm{Cl})\end{array}$} \\
\hline & $\mathrm{N}$ & $\%$ & $\mathrm{~N}$ & $\%$ & \\
\hline cats strictly kept inside & 33 & 23.6 & 0 & 0 & $1 *$ \\
\hline free-roaming cats & 50 & 35.7 & 5 & 10 & $7.3(0.41-128.35)$ \\
\hline stray cats & 28 & 20 & 10 & 35,7 & $24.6(1.50-402.25)$ \\
\hline $\begin{array}{l}\text { cats from shelters and } \\
\text { deposits }\end{array}$ & 29 & 20.7 & 9 & 31 & $21.5(1.30-354.52)$ \\
\hline$\Sigma$ & 140 & 100 & 24 & 17,4 & \\
\hline
\end{tabular}

*1 $=$ reference group

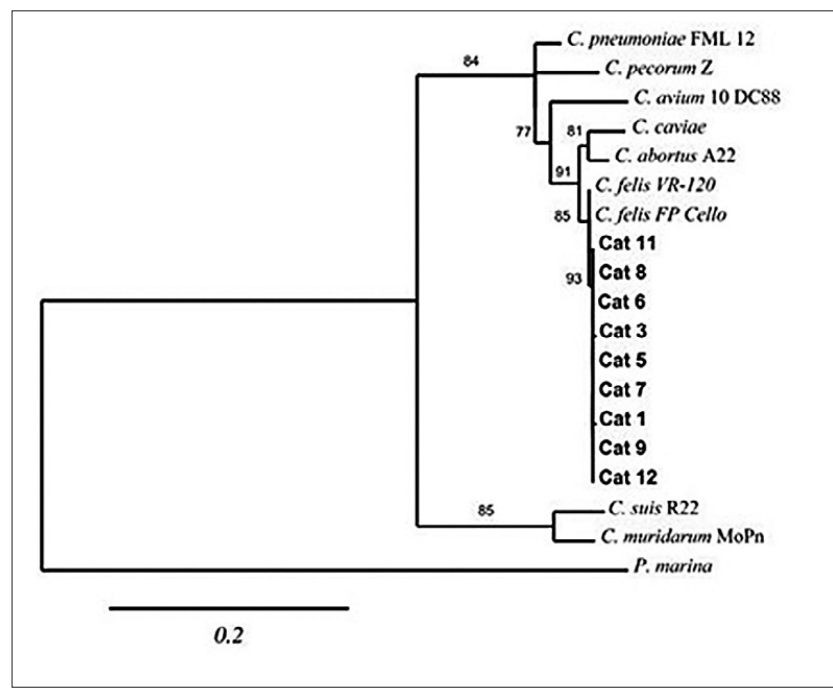

Figure 1. Phylogenetic tree of $C$. felis isolates from the presented study (cat) and selected accessions of chlamydial species from GenBank, based on 23S rRNA gene fragment sequences

\section{DISCUSSION AND CONCLUSIONS}

With the growing interest in the breeding and adoption of cats from shelters in Slovakia, the question arises about the prevalence of certain infectious diseases of cats. Conjunctivitis and respiratory diseases are frequently occurring clinical symptoms in young stray cats [18]. One of the major pathogens in cats is Chlamydia felis, which is a bacterial agent of upper respiratory tract infection and conjunctivitis.

C. felis is endemically spread between domestic cats worldwide, and its prevalence has been published in several studies [7, 19, 20, 21, 22, 23]. A 17.1\% prevalence of C. felis infection was found Slovakia, diagnosed by the PCR method. Of the total number of healthy cats $(\mathrm{n}=71), C$. felis infection only in 3 cases, representing a $4.2 \%$ prevalence of chlamydiosis in healthy cats. This result correlates with studies published in other countries. Prevalence of $C$. felis in clinicaly healthy cats in Italy is 3.3\% [24] and in the USA - 0\% [25]. In clinically healthy animals from several European pedigree catteries or shelters, the prevalence of C. felis was 3\% [21].

In the presented study, the prevalence of $C$. felis infection in the population of cats showing clinical signs was $30.4 \%$. The relative risk of the disease is 7 times higher $(\mathrm{P}=0.0095)$ in cats with clinical signs of the disease than in cats without clinical signs.

Feline chlamydiosis is one of the most common disease in cats, but there is very little information regarding the prevalence of the disease in Slovakia. Prevalence of C. felis in Slovakia confirmed by IFA has been published previously [22]. In the current study, the total prevalence of C. felis in cats with clinical signs of conjunctivitis was $45.16 \%$, with the highest proportion of positive cats comimg from shelters (65.8\%). Since there is a lack of studies focused on screening for capturing the most common pathogens of conjunctiva and upper respiratory tract in Slovakia, proportion of $C$. felis pathogen in total infections in these inseparable systems in cats remains unclear.

In other studies, the prevalence of $C$. felis in cats with upper respiratory tract disease determined by culture is in the range of $23-31 \%[26,27]$. Studies using PCR of 
samples from conjunctiva led to the specification of the prevalence of $14.3 \%(66 / 462)$ in cats with upper respiratory tract disease (URTD) [28]; 17.7\% (20/113) in cats with symptoms of conjunctivitis [29]; 20\% (14/70) in cats also with conjunctivitis [20], and 11.5\% (26/226) in Swiss cats with eye symptoms [30]. Differences in prevalence may be due to different diagnostic methods. It seems that the significance of C. felis as a causative agent of URTD and conjunctiva differ among countries. While in the USA this agent is considered less important than feline herpesvirus 1 (FHV1) [31], C. felis is the most common pathogen causing conjunctivitis and URTD in Japan [32]. An explanation may be the difference in the prevalences in these countries.

In the current study, almost all the infected cats had clinical symptoms of conjunctivitis. The most common clinical symptom of conjunctivitis was purulent discharge from the eyes, and affected $50 \%$ of the infected cats. Some literary sources report an increased incidence of chemosis and less severe mucosal discharge $[8,18]$. However, not all affected cats show symptoms of conjunctivitis, and therefore the absence of clinical signs does not indicate the absence of infection. Sparkes et al. (1999) [33] reported that the clinical symptoms may subside in untreated cats within 3 months, but the excretion of $C$. felis can continue for at least 8 months. The prevalence of $C$. felis in asymptomatic cats is low. In studies using PCR was $<5 \%[20,24,28,30]$. Persistent excretion of chlamydia after subsidence of clinical signs may be an explanation for the positivity in some asymptomatic cats [30].

Several risk factors for this infection have been described, especially the impact of the environment in which cats live, the concentration of animals in the room and zoohygienic conditions in breeding farms and animal shelters. Previous studies show that up to $95 \%$ of infected cats came from shelters with poor zoohygienic conditions [21]. Also, an important risk factor in the transmission of infection is the presence of other animals. Studies by Binns et al. (2000) [34] and Dawson et al. (2000) [35] have shown that a significant proportion of the incidence of upper respiratory tract disease may be played by the presence of dogs. A potential source of infection of cats are also birds and rodents [36, 37]. The disadvantage of deposit centres is that they often have other kinds of animals, such as dogs and exotic birds.

In the current study, $0 \%$ prevalence was found in household cats kept strictly indoors. In the population of free-roaming domestic cats, which have the possibility of contact with other cats and animals, the prevalence was $10 \%$, which is comparable to the $11.5 \%$ prevalence in Australia [7]. GruffydJones et al. [9] found in their study the highest prevalence of chlamydiosis in breeding farms and shelters. The prevalence in shelters and stray cats, according to the current study, was similar and represents $31 \%$ and $35.7 \%$, respectively. In another study by Helps et al. (2005) [21] who studied 1,748 cats from 218 shelters in different European countries, found that C. felis was at the level of $10 \%$ in affected cats and $3 \%$ in healthy cats. Similarly, other epidemiological studies found differences in the incidence of $C$. felis infection in cats, depending on the farming method. In a study that analyzed a shelter with a high density of cats of unknown origin, and had a history of upper respiratory tract infection and acute and chronic conjunctivitis, a high incidence $(58.06 \%)$ of $C$. felis in the cats was found in comparison with previous studies. These data suggest that a high density of animals may be associated with the spread of the agents. All cats in this the study had clinical signs of upper respiratory tract infection, and most cats positive for $C$. felis had a mucopurulent discharge from the eyes or nasal secretion [38]. These differences are likely to be attributed to the lifestyle and animal welfare.

The most important reservoirs of chlamydia, mainly C. psittaci, are feral pigeons which are widespread in several European cities and towns. The prevalence in these birds ranged from $1.6 \%-95.6 \%[39,40,41,42]$. Stray cats have more opportunities to interact with infected birds or other animals, and suffer from inadequate nutrition and possibly weakened immune systems that can contribute to increased exposure to infectious pathogens. This may explain the higher prevalence of $C$. felis in stray cats than in cats kept strictly indoors.

This also raises the issue non-existent shelters for cats in Slovakia. At present, the problem of catching and placing the cats is solved through the so-called deposit centres. The owners of these „shelters“are private individuals who are trying to cooperate with a non-profit organization to help tackle the issue of adopting cats from the streets, thereby reducing the number of cats and their total population. Cats are held at these deposit centres/shelters until owners are found for them which, in some cases, may take several months or even years. The arrival of other new animals in such an environment causes the mutual exchange of various types of infections, including chlamydia, and becomes a reservoir of diseases for the healthy cats. The starting point of a poorly-funded deposit centre and a lack of awareness by the temporary owners of the cats, is the death rate, often affecting up seversal cats among a high concentration of animals. By complying with the principles of zoohygiene, quarantine, therapy and vaccination of animals in shelters and breeding colonies, the incidence of the disease should be minimized. Based on the identified risk groups, the vaccination of the cats is recommend. Live attenuated vaccines or inactivated vaccines are available, but their use should be restricted to animals exclusively at risk.

Zoonotic risk of $C$. felis is known and its transmission to humans has been documented in several studies $[10$, $14,43,44]$. In humans, C. felis may cause conjunctivitis, and/or respiratory tract diseases, [10], pneumonia [11], hepatosplenomegaly, glomerulonephritis and endocarditis, especially in those who live in close contact with cats [12]. They, may also cause abortion, neonatal mortality and infertility [13]. However, as a study of seroprevalence between cats and their owners has shown, the risk of infection is very low [6].

The obtained results show a high proportion of C. felis in the etiology of infections of the conjunctiva and upper respiratory tract, and an endemic presence of this pathogen in the population of stray and shelter cats. The probability of the infection in cats that have no possibility of contact with other cats and animals is very low, and the same is the case of animals without clinical signs of disease. The risk of the disease in cats with clinical signs of infection is 7 times higher than in cats with no clinical symptoms, but it can also be found in clinicaly healthy cats.

Cats could play an important role in the transmission of chlamydiosis to humans. By understanding and following the principles of hygiene, quarantine, treatment and vaccination of animals in shelters and breeding colonies, the occurrence of the infection could be decreased.

\section{Acknowledgements}

The study was supported by the Slovak Grant Committee Nos.: VEGA No. 1/0084/18 and APVV 15-0134. 


\section{REFERENCES}

1. Taylor LH, Latham SM, Woolhouse ME. Risk factors for human disease emergence. Philos Trans R Soc Lond B Biol Sci. 2001; 356(1411): 983-989.

2. Alemayehu A. Review on Emerging and Re-Emerging Bacterial Zoonotic Diseases. Am-Euras J Sci Res. 2012; 7(4): 176-186.

3. Vorimore F, Hsia RC, Huot-Creasy H, Bastian S, Deruyter L, Passe A, et al. Isolation of a New Chlamydia species from the Feral Sacred Ibis (Threskiornis aethiopicus): Chlamydia ibidis. PLoS One. 2013; 8(9): e74823.

4. Sachse K, Bavoil PM, Kaltenboeck B, Stephens RS, Kuo CH, RossellóMora R, et al. Emendation of the family Chlamydiaceae: Proposal of a single genus, Chlamydia, to include all currently recognized species. Syst Appl Microbiol. 2015; 38(2): 99-103.

5. TerWee J, Sabara M, Kokjohn K, Sandbulte J, Frenchick P, Dreier KJ. Characterization of the systemic disease and ocular signs induced by experimental infection with Chlamydia psittaci in cats. Vet Microbiol 1998; 59(4): 259-281.

6. Yan C, Fukushi H, Matsudate H, Ishihara K, Yasuda K, Kitagawa H, et al. Seroepidemiological investigation of feline chlamydiosis in cats and humans in Japan. Microbiol Immunol. 2000; 44(3): 155-160.

7. Sykes JE. Feline upper respiratory tract pathogens: Chlamydophila felis. Comp Contin Educ Pract Vet. 2001; 23(3): 231-240.

8. Sykes JE. Feline chlamydiosis. Clin Tech Small Anim Pract. 2005; 20(2): 129-134.

9. Gruffydd-Jones T, Addie D, Belák S, Boucraut-Baralon C, Egberink $\mathrm{H}$, Frymus T, et al. Chlamydophila felis infection. ABCD guidelines on prevention and management. J Fel Med Surg. 2009; 11(7): 605-609.

10. Corsaro D, Venditti D, Valassina M. New parachlamydial 16S rDNA phenotypes detected in human clinical samples. Res Microbiol. 2002; 153(9): 563-567.

11. Marrie TJ, Peeling RW, Reid T, De Carolis E. Chlamydia species as a cause of community-acquired pneumonia in Canada. Eur Respir J. 2003; 21(5): 779-784.

12. Griffiths PD, Lechler RI, Treharne JD. Unusual chlamydial infection in a human renal allograft recipient. Br Med J. 1978; 2(6147): 1264-1265.

13. Pointon A M, Nicholls JM, Neville S. Chlamydia infection among breeding catteries in South Australia. Aust Vet Practit. 1991; 21: 58-63.

14. Hartley JC, Stevenson S, Robinson AJ, Littlewood JD, Carder C, Cartledge J, et al. Conjunctivitis due to Chlamydophila felis (Chlamydia psittaci feline pneumonitis agent) acquired from a cat: case report with molecular characterization of isolates from the patient and cat. J Infect. 2001; 43(1): 7-11.

15. Azari AA, Barney NP. Conjunctivitis: a systematic review of diagnosis and treatment. JAMA. 2013; 310(16): 1721-1729.

16. Azuma Y, Hirakawa H, Yamashita A, Cai Y, Rahman MA, Suzuki H et al. Genome sequence of the cat pathogen, Chlamydophila felis. DNA Res. 2006; 13(1): 15-23.

17. Basic Local Alignment Search Tool. NCBI National Center for Biotechnology Information. https://blast.ncbi.nlm.nih.gov/Blast. cgi?PROGRAM=blastn\&PAGE_TYPE =BlastSearch\&LINK_ LOC=blasthome (accsess 2018.05.10)

18. Gelatt KN. Veterinary ophthalmology. 4th ed. Lippincott Wiliams and Wikins, Pensylvania, USA; 2007. p. 1107-1154.

19. Cai Y, Fukushi H, Koyasu S, Kuroda E, Yamaguchi T, Hirai K. An etiological investigation of domestic cats with conjunctivitis and upper respiratory tract disease in Japan. J Vet Med Sci. 2002; 64(3): 215-219.

20. Rampazzo A, Appino S, Pregel P, Tarducci A, Zini E, Biolatti B. Prevalence of Chlamydophila felis and feline herpesvirus 1 in cats with conjunctivitis in northern Italy. J Vet Intern Med. 2003; 17(6): 799-807.

21. Helps CR, Lait P, Damhuis A, Björnehammar U, Bolta D, Brovida C et al. Factors associated with upper respiratory tract disease caused by feline herpesvirus, feline calicivirus, Chlamydophila felis and Bordetella bronchiseptica in cats: experience from 218 European catteries. Vet Rec. 2005; 156(21): 669-673.

22. Halánova M, Sulinova Z, Čislákova L, Trbolová A, Páleník L, Weissová $\mathrm{T}$, et al. Chlamydophila felis in cats-are the stray cats dangerous source of infection? Zoonos Pub Health. 2011; 58(7): 519-522.

23. Wielieczko AK, Ploneczka-Janeczko K. Feline herpesvirus 1 and Chlamydophila felis prevalence in cats with chronic conjunctivits. Pol J Vet Sci. 2010; 13(2):381-383.

24. Di Francesco A, Piva S, Baldelliw R. Prevalence of Chlamydia felis by PCR among healthy pet cats in Italy. New Microbiol. 2004; 27(2): 199-202.

25. Low HC, Powell CC, Veir JK, Hawley JR, Lappin MR. Prevalence of feline herpesvirus 1, Chlamydophila felis, and Mycoplasma spp DNA in conjunctival cells collected from cats with and without conjunctivitis Am J Vet Res. 2007; 68(6): 643-648.

26. Wills JM, Howard PE, Gruffydd-Jones TJ, Wathes CM. Prevalence of Chlamydia psittaci in different cat populations in Britain. J Small Anim Pract. 1988; 29(6): 327-339.

27. Hanselaer JR, Derore A, Boucherie P. Demonstration of Chlamydia psittaci in feline conjunctivitis cases in Belgium Vlaams Diergeneeskd Tijdschr. 1989; 58: 165-168.

28. Sykes JE, Anderson GA, Studdert VP, Browning GF. Prevalence of feline Chlamydia psitaci and feline herpesvirus 1 in cats with upper respiratory tract disease. J Vet Intern Med. 1999; 13(3): 153-162.

29. McDonald M, Willett BJ, Jarrett O, Addie DD. A comparison of DNA amplification, isolation and serology for the detection of Chlamydia psittaci infection in cats. Vet Rec. 1998; 143(4): 97-101.

30. von Bomhard W, Polkinghorne A, Lu ZH, Vaughan L, Vögtlin A, Zimmermann DR, et al. Detection of novel Chlamydiae in cats with ocular disease. Am J Vet Res. 2003; 64(11): 1421-1428.

31. Veir JK, Ruch-Gallie R, Spindel ME, Lappin MR. Prevalence of selected infectious organisms and comparison of two anatomic sampling sites in shelter cats with upper respiratory tract disease. J Feline Med Surg. 2008; 10(6): 551-557.

32. Iwamoto K, Masubuchi K, Nosaka H, Kokubu T, Nischida K, Toshida $\mathrm{T}$, et al. Isolationa of Chlamydia psittaci from domestic cats with oculonasal discharge in Japan. J. Vet. Med. Sci. 2001; 63(8): 937-938.

33. Sparkes AH, Caney SM, Sturgess CP, Gruffydd-Jones TJ. The clinical efficacy of topical and systemic therapy for the treatment of feline ocular chlamydiosis. J Feline Med Surg. 1999; 1(1): 31-35.

34. Binns SH, Dawson S, Speakman AJ, Cuevas LE, Hart CA, Gaskell CJ, et al. A study of feline upper respiratory tract disease with reference to prevalence and risk factors for infection with feline calicivirus and feline herpesvirus. J Feline Med Surg. 2000; 2(3): 123-133.

35. Dawson S, Gaskell CJ, McCracken CM, Gaskell RM, Hart CA, Jones D. Bordetella bronchiseptica infection in cats following contact with infected dogs. Vet Rec. 2000; 146(2): 46-48.

36. Čisláková L, Stanko M, Fričová J, Mošanský L, Trávniček M, Halánová $\mathrm{M}$, et al. Small mammals (Insectivora, Rodenteria) as a potentional source of chlamydial infection in east Slovakia. Ann Agric Environ Med. 2004; 11(1): 139-143.

37. Takáčová D, Halánová M, Čisláková L, Kováčová D, Halán M, Jarčuška P. Long-term monitoring for ornithosis - Psittacosis and mammals chlamydiosis in animals in Slovakia. Acta Vet-Beograd. 2010; 60(2-3): 241-248.

38. Gonsales FF, Brandão PE, Melville PA, da Hora AS, Zuniga E, Saidenberg A, et al. Occurrence of Chlamydophila felis in a cattery in Osasco city, São Paulo state. Braz. J Vet Res Anim Scien. 2013; 50(5): 379-383.

39. Pruckner-Radovcic E, Horvatek D, Gottstein Y, Grozdanic IC, Mazija H. Epidemiological investigation of Chlamydophila psittaci in pigeons and free living birds in Croatia. Vet Res Commun. 2005; 29: 17-21.

40. Ceglie L, Lafisca S, Guadagno C, Dalla Pozza G, Capello K, Bano L, et al. Serological surveillance in north-eastern Italy for the presence of Chlamydophila spp. from birds and molecular characterisation of PCR isolates within the area of Venice. Proceedings of the 5th Annual Workshop of COST Action 855 "Animal chlamydioses and its zoonotic implications”, Pulawy (Poland), September. 2007; 10-11: 62-67.

41. Vlahovič K, Matica B, Bata I, Pavlak M, Pavičič Ž, Popovič M, et al. Campylobacter, salmonella and chlamydia in freeliving birds of Croatia. Eur J Wildl Res. 2004; 50: 127-132.

42. Haag-Wackernagel D. Human diseases caused by feral pigeons. In: Chris, J., Feare, David, P., Cowan, (Eds.), Advances in Vertebrate Pest Management 4. Filander Verlag, Fürth, 2006; 31-58.

43. Ostler HB, Schachter J, Dawson CR. Acute follicular conjuctivitis of epizootic origin. Feline pneumontis. Arch Ophthalmol. 1969; 82(5): 587-591.

44. Miyashita N, Fukano H, Mouri K, Fukuda M, Yoshida K, Kobashi $\mathrm{Y}$, et al. Community-acquired pneumonia in Japan: a prospective ambulatory and hospitalized patient study. J Med Microbiol. 2005; 54(4): 395-400.

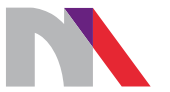

Ministry of Science and Higher Education

Republic of Poland

Generation of the DOI (Digital Object Identifier) - task financed under the agreement No. 618/P-DUN/2019 by the Minister of Science and Higher Education 